\title{
HOLOMORPHIC MAPS THAT EXTEND TO AUTOMORPHISMS OF A BALL
}

\author{
WALTER RUDIN ${ }^{1}$
}

\begin{abstract}
ABSTRACr. It is proved, under hypotheses that may be close to minimal, that certain types of biholomorphic maps of subregions of the unit ball in $\mathbf{C}^{n}$ have the extension property to which the title alludes.
\end{abstract}

Let $B$ (or $B_{n}$, when necessary) denote the open unit ball of $\mathbf{C}^{n}$. Thus $z=$ $\left(z_{1}, \ldots, z_{n}\right) \in B$ provided that $|z|\left\langle 1\right.$, where $|z|=\langle z, z\rangle^{1 / 2}$ and $\langle z, w\rangle=\sum z_{j} \bar{w}_{j}$. An automorphism of $B$, i.e., a member of $\operatorname{Aut}(B)$, is, by definition, a holomorphic map of $B$ onto $B$ that is one-to-one, and whose inverse is therefore also holomorphic. The sphere that bounds $B$ is denoted by $S$.

The following extension theorem will be proved.

THEOREM. Assume that $n>1$, and that

(a) $\Omega_{1}$ and $\Omega_{2}$ are connected open subsets of $B$,

(b) for $j=1,2, \Gamma_{j}$ is an open subset of $S$ such that $\Gamma_{j} \subset \partial \Omega_{j}$,

(c) $F$ is a holomorphic one-to-one map of $\Omega_{1}$ onto $\Omega_{2}$, and

(d) there is a point $\alpha \in \Gamma_{1}$, not a limit point of $B \cap \partial \Omega_{1}$, and a sequence $\left\{a_{i}\right\}$ in $\Omega_{1}$, converging to $\alpha$, such that $\left\{F\left(a_{i}\right)\right\}$ converges to a point $\beta \in \Gamma_{2}$, not a limit point of $B \cap \partial \Omega_{2}$.

Then there exists $\Phi \in \operatorname{Aut}(B)$ such that $\Phi(z)=F(z)$ for all $z \in \Omega_{1}$.

The relation of this theorem to earlier results will be discussed after its proof.

The proof will use the following well-known facts.

(I) If $F: B_{k} \rightarrow B_{n}$ is holomorphic, and $F(0)=0$, then $|F(z)<| z \mid$ for all $z \in B_{k}$, and the linear operator $F^{\prime}(0)$ (the Fréchet derivative of $F$ at 0 ) maps $B_{k}$ into $B_{n}$.

(II) If, in addition, $k=n$, then the Jacobian $J F$ of $F$ satisfies $|(J F)(0)|<1$; equality holds only when $F$ is a unitary operator on $\mathbf{C}^{n}$.

(III) If $F \in \operatorname{Aut}(B)$ and $F(0)=0$, then $F$ is unitary.

Here is a brief indication of how these are proved. For unit vectors $u$ and $v$ in $\mathbf{C}^{k}$ and $\mathbf{C}^{n}$, respectively, the classical Schwarz lemma applies to the function $g$ defined by

$$
g(\lambda)=\langle F(\lambda u), v\rangle, \quad(\lambda \in \mathbf{C},|\lambda|<1) .
$$

Received by the editors January 8, 1980.

AMS (MOS) subject classifications (1970). Primary 32D15.

${ }^{1}$ This research was partially supported by NSF Grant MCS 78-06860, and by the William F. Vilas Trust Estate. 
Thus $|g(\lambda)|<|\lambda|$ for all eligible $u$, $v$, which leads to $|F(z)|<|z|$, and $\left|g^{\prime}(0)\right|<1$, which completes $(\mathrm{I})$, since

$$
g^{\prime}(0)=\left\langle F^{\prime}(0) u, v\right\rangle .
$$

Since (I) implies that no eigenvalue of $F^{\prime}(0)$ exceeds 1 in absolute value, it follows that

$$
|(J F)(0)|=\left|\operatorname{det} F^{\prime}(0)\right|<1 .
$$

If $|(J F)(0)|=1$, then the linear operator $F^{\prime}(0)$ preserves volume, and maps $B$ into $B$, hence is a unitary operator $U$. From this it follows easily (by considering iterates of $U^{-1} F$ ) that $F=U$.

To prove (III), apply (II) to $F$ as well as to $F^{-1}$.

The following lemma contains the essence of the proof of the theorem. To state it, we introduce the notation (for $z \in \mathbf{C}^{\boldsymbol{n}}$ )

$$
D_{z}=\{\lambda z: \lambda \in \mathbf{C}, \lambda z \in B\} .
$$

Thus, when $z \neq 0, D_{z}$ is the disc that is the intersection with $B$ of the complex line through 0 and $z$.

Lemma. Assume that

(i) $\Omega_{1}$ and $\Omega_{2}$ are connected open sets in $B$,

(ii) $0 \in \Omega_{1}, 0 \in \Omega_{2}$.

(iii) $F$ is a holomorphic one-to-one map of $\Omega_{1}$ onto $\Omega_{2}$, with $F(0)=0$, and

(iv) there is a nonempty open set $V \subset \Omega_{1}$, such that $D_{z} \subset \Omega_{1}$ and $D_{F(z)} \subset \Omega_{2}$ for every $z \in V$.

Then there is a unitary transformation $U$ on $\mathbf{C}^{n}$ such that $F(z)=U z$ for all $z \in \Omega_{1}$.

Proof of the Lemma. If $z \in V$, then $D_{z}$ lies in the domain of $F$. Identifying $D_{z}$ with $B_{1}$, we see from fact (I) (the case $k=1$ ), that $|w|<|z|$, where $w=F(z)$. But $D_{w}$ lies in the domain of $F^{-1}$, and the same argument shows that $|z|<|w|$. Thus $|F(z)|^{2}=|z|^{2}$ for all $z \in V$. Both of these functions are real-analytic, hence they are equal in all of $\Omega_{1}$. In particular, choosing $r>0$ so small that $r B \subset \Omega_{1}$, we see that $|F(z)|=|z|$ for all $z \in r B$. An appropriately scaled version of fact (III) shows now that $F$ is unitary.

Proof of The Theorem. Let $\left\{a_{i}\right\}$ be as in assumption (d), put $b_{i}=F\left(a_{i}\right)$, and choose $u_{i} \in S, v_{i} \in S$, so that

$$
a_{i}=\left|a_{i}\right| u_{i}, \quad b_{i}=\left|b_{i}\right| v_{i}, \quad(i=1,2,3, \ldots) .
$$

The geometric information contained in (d) shows that there exists $t<1$ such that, setting

$$
E_{t}(\xi)=\{z \in B: t<\operatorname{Re}\langle z, \xi\rangle\}, \quad(\xi \in S),
$$

we have $a_{i} \in E_{t}\left(u_{i}\right) \subset \Omega_{1}$, and $b_{i} \in E_{t}\left(v_{i}\right) \subset \Omega_{2}$ for all sufficiently large $i$, say $i>i_{0}$.

If $a \in B \backslash\{0\}$, let $P$ denote the orthogonal projection of $\mathbf{C}^{\boldsymbol{n}}$ onto the one-dimensional subspace spanned by $a$, put $Q=I-P$, and define

$$
\varphi_{a}(z)=\frac{a-P z-\left(1-|a|^{2}\right)^{1 / 2} Q z}{1-\langle z, a\rangle}, \quad(z \in \bar{B}) .
$$


Then (see [4], for instance) $\varphi_{a} \in \operatorname{Aut}(B)$ and $\varphi_{a}^{-1}=\varphi_{a}$. Define

$$
G_{i}=\varphi_{b_{i}} \circ F \circ \varphi_{a_{i}}, \quad\left(i>i_{0}\right) .
$$

Each $G_{i}$ is a holomorphic one-to-one map of $\Omega_{1}^{i}=\varphi_{a}\left(\Omega_{1}\right)$ onto $\Omega_{2}^{i}=\varphi_{b_{1}}\left(\Omega_{2}\right)$, and $G_{i}(0)=0$.

If $a=|a| \xi$, then $\langle P z, \xi\rangle=\langle z, \xi\rangle \xi$, hence

$$
\left\langle\varphi_{a}(z), \xi\right\rangle=(|a|-\langle z, \xi\rangle) /(1-|a|\langle z, \xi\rangle) .
$$

If $t<|a|$, it follows that $\varphi_{a}\left(E_{t}(\xi)\right)$ contains all $z \in B$ with

$$
\operatorname{Re}\langle z, \xi\rangle<(|a|-t) /(1-|a| t) \text {. }
$$

Since $\left|a_{i}\right| \rightarrow 1$ and $\left|b_{i}\right| \rightarrow 1$, and since the right side of (10) tends to 1 as $|a|$ tends to 1 , there is a sequence $\left\{r_{i}\right\}, r_{i}<1$, such that $r_{i} \rightarrow 1$ as $i \rightarrow \infty$, and such that

$$
\begin{aligned}
& z \in B, \operatorname{Re}\left\langle z, u_{i}\right\rangle\left\langle r_{i} \text { implies } z \in \Omega_{1}^{i},\right. \\
& w \in B, \operatorname{Re}\left\langle z, v_{i}\right\rangle\left\langle r_{i} \text { implies } w \in \Omega_{2}^{i} .\right.
\end{aligned}
$$

By (11), $r_{i} B \subset \Omega_{1}^{i}$, the domain of $G_{i}$. Since $G_{i}(0)=0$, fact (II) gives $\left|\left(J G_{i}\right)(0)\right|<$ $r_{i}^{-n}$. In the same way, (12) leads to $\left|\left(J G_{i}^{-1}\right)(0)\right|<r_{i}^{-n}$, so that $\left|\left(J G_{i}\right)(0)\right|>r_{i}^{n}$. A normal family argument shows now that a subsequence of $\left\{G_{i}\right\}$ converges, uniformly on compact subsets of $B$, to a holomorphic map of $B$ into $B$ that fixes 0 and whose Jacobian at 0 has absolute value 1 . By fact (II), this limit map is unitary. Call it $U$.

Let $V_{i}$ be the set of all $p \in B$ such that

$$
D_{z} \subset \Omega_{1}^{i} \text { and } D_{U_{z}} \subset \Omega_{2}^{i}
$$

for all $z$ in some neighborhood of $p$.

Now fix $\varepsilon, 0<\varepsilon<1 / 10$. Using (11)-(13), we see that there is an index $i$, fixed from now on, such that

$$
\left|G_{i}(z)-U z\right|<\varepsilon \quad \text { whenever }|z|<1-\varepsilon,
$$

and such that $V_{i}$ contains a ball of radius $2 \varepsilon$, whose center $p$ satisfies $|p|<1-3 \varepsilon$. To see in more detail that this can indeed be done, note that when $r_{i}$ is sufficiently close to 1 , there exists a large set of points $\xi \in S$ such that $\left|\left\langle\xi, u_{i}\right\rangle\right|\left\langle r_{i}\right.$ and $\left|\left\langle\xi, U^{-1} v_{i}\right\rangle\right|<r_{i}$. For any such $\xi, D_{\xi} \subset \Omega_{1}^{i}$ and $D_{U \xi} \subset \Omega_{2}^{i}$, thus $\lambda \xi \in V_{i}$ if $0<|\lambda|<$ 1 .

Thus $D_{z} \subset \Omega_{1}^{i}$ if $|z-p|<2 \varepsilon$, and $D_{w} \subset \Omega_{2}^{i}$ if $|w-U p|<2 \varepsilon$. If $|z-p|<\varepsilon$, and $w=G_{i}(z)$, it follows that $D_{w} \subset \Omega_{2}^{i}$ because

$$
|w-U p|<\left|G_{i}(z)-U z\right|+|z-p|<2 \varepsilon .
$$

The lemma applies therefore to $G_{i}$ and shows that $G_{i}$ is (the restriction of) a unitary operator. Since (8) gives

$$
F=\varphi_{b_{i}} \circ G_{i} \circ \varphi_{a_{i}},
$$

the theorem is proved.

REMARKS. (i) Let $\Omega$ be a connected open subset of $B$ such that $\bar{\Omega}$ contains an open subset $\Gamma$ of $S$. If $F$ is a nonconstant $C^{1}$-map of $\bar{\Omega}$ into $\bar{B}$ that is holomorphic in $\Omega$ and carries $\Gamma$ into $S$, then $F \in \operatorname{Aut}(B)$. This was proved by Pinčuk [6, p. 381], 
who extended an earlier version due to Alexander [1] in which $C^{\infty}$ was assumed in place of $C^{1}$.

This Alexander-Pinčuk result is a fairly direct corollary of the present theorem. If $F \in C^{1}(\bar{\Omega})$ satisfies the Alexander-Pinčuk hypotheses, it is not hard to show (see Fornaess [3, p. 549] or Pinčuk [6, p. 378]) that $J F$ vanishes at no point of $\Gamma$. The inverse function theorem implies then that the hypotheses of the present theorem hold.

(ii) In Alexander's proof [2] that every proper holomorphic map of $B$ into $B$ is in Aut $(B)$ when $n>1$, his appeal to Fefferman's theorem can be replaced by the one proved in the present paper. Consequently, there exists now a much more elementary proof of the proper mapping theorem for $B$.

(iii) It is quite possible that the present theorem remains true if $B$ is replaced by strictly pseudoconvex domains with real-analytic boundaries (as Pinčuk did in the $C^{1}$-case [7]), but an entirely different proof would have to be found; Rosay [8] (strengthening a result of Wong [9]) proved that if some boundary point $\xi$ of a bounded domain $\Omega \subset \mathbf{C}^{n}$ is a point of strict pseudoconvexity, and if there exist automorphisms $T_{k}$ of $\Omega$ such that $\lim _{k \rightarrow \infty} T_{k}(p)=\xi$ for some $p \in \Omega$, then $\Omega$ is biholomorphically equivalent to $B$.

In other strictly pseudoconvex bounded domains there are thus insufficiently many automorphisms to imitate the proof that works in $B$.

(iv) If $\xi \in S$ and $\Omega=B \cap\{z:|\xi-z|<1\}$; in other words, if $\Omega=B \cap(\xi+B)$, then the map $z \rightarrow \xi-z$ of $\Omega$ onto $\Omega$ demonstrates the relevance of the assumptions concerning the location of the points $\alpha$ and $\beta$ in our theorem.

\section{REFERENCES}

1. H. Alexander, Holomorphic mappings from the ball and polydisc, Math. Ann. 209 (1974), 249-256.

2. __ Proper holomorphic mappings in Cn, Indiana Univ. Math. J. 26 (1977), 137-146.

3. J. E. Fornaess, Embedding strictly pseudoconvex domains in convex domains, Amer. J. Math. 98 (1976), 529-569.

4. A. Nagel and W. Rudin, Moebius-invariant function spaces on balls and spheres, Duke Math. J. 43 (1976), 841-865.

5. S. I. Pinčuk, On proper holomorphic mappings of strictly pseudoconvex domains, Siberian Math. J. 15 (1974), 644-649.

6. __ On the analytic continuation of holomorphic mappings, Math. USSR-Sb. 27 (1975), 375-392.

7. Analytic continuation of mappings along strictly pseudoconvex hypersurfaces, Soviet Math. Dokl. 18 (1977), 1237-1240.

8. J. P. Rosay, Sur une caractérisation de la boule parmi les domaines de $\mathrm{C}^{\mathbf{n}}$ par son groupe d'automorphismes, Ann. Inst. Fourier 29 (1979), 91-97.

9. B. Wong, Characterization of the unit ball in C' by its automorphism group, Invent. Math. 41 (1977), 253-257.

Department of Mathematics, University of Wisconsin-Madison, Madison, Wisconsin 53706 